\title{
FACTORES PERSONALES, DE GÉNERO Y SOCIOECONÓMICOS RELACIONADOS CON EL CYBERBULLYING EN ADOLESCENTES COLOMBIANOS
}

\author{
William Fernando Morales Portilla, Ángela Yamile Rueda Forero, Jesús Redundo Pacheco, Marianela \\ Luzardo y Nataly Gómez Correa \\ Universidad Pontificia Bolivariana \\ Colombia
}

\begin{abstract}
RESUMEN
La violencia escolar se ha nutrido de las tecnologías de la información y de la comunicación (TIC) para desarrollar nuevas dinámicas violentas, entre ellas el ciberbullying. Este fenómeno es cada vez más preocupante por su alcance, ya que en la población donde surge con mayor proporción es en adolescentes (Luengo, 2014). De esta manera, la presente investigación con un paradigma analítico de tipo transversal-exploratorio tuvo como objetivo determinar si las variables como el estrato socioeconómico, género y la personalidad representan un factor de riesgo en la presentación del cyberbullying en estudiantes de secundaria con edades de entre 10 y 19 años. Se utilizó una metodología cuantitativa, donde la muestra estuvo conformada por 404 estudiantes de secundaria de dos centros educativos de Bucaramanga, Colombia, con edades de entre 10 y 19 años, de los que $49.5 \%$ son varones y $50.5 \%$ mujeres. Para el logro de los objetivos se aplicó el cuestionario de Cyberbullying de Calmaestra, Ortega y Mora-Merchán, el cuestionario de personalidad PPG-IPG de Gordon, y un cuestionario sociodemográfico que permitió evaluar las variables de estrato socioeconómico y de género. Los resultaron demostraron que existen relaciones estadísticamente significativas entre el ciberacoso realizado por teléfono celular o internet y los estratos del 1 al 5; además de relaciones estadísticamente significativas entre el género masculino y ser cibervíctima por internet. Del mismo modo, se pudo evidenciar una relación directa entre el género masculino y ser ciberagresor por medio de internet y el teléfono celular. Como conclusión, se destaca la importancia de seguir desarrollando procesos investigativos referentes al cyberbullying, resaltando la variable de estrato socioeconómico, porque esta distribución por zonas económicas es propia de países en vía de desarrollo.
\end{abstract}

Palabras Clave:

cyberbullying, factores de riesgo, género, estrato socioeconómico, rasgos de personalidad.

\section{PERSONAL, GENDER AND SOCIOECONOMIC FACTORS RELATED TO CYBERBULLYING IN COLOMBIAN ADOLESCENTS}

\begin{abstract}
School violence has been increasing by the TIC (short for Information and Communication Technologies in Spanish) to develop new dynamics of violence, Cyberbullying among them. This issue is more and more worrying due to its wide range which can reach teenagers in a bigger proportion (Luengo, 2014). Thence, this investigation with an analytical paradigm of transversal-exploratory type had as objective to determine if variables as socioeconomic stratum, genre and personality features represent any factor of risk in the cyberbullying. A quantitative methodology was used, the sample was formed by 404 high-school students from two schools in Bucaramanga, Colombia with ages between 10 and 19 years old of whom $49.5 \%$ are male and $50.5 \%$ are female. To accomplish the objectives, we applied a survey about cyberbullying from Calmaestra, Ortega y Mora-Merchán, a questionnaire about personality called PPG-IPG (short for Gordon's Personal Profile and Gordon's Personal Inventory), and a sociodemographic questionnaire, which permitted evaluate the socioeconomic stratum and gender variables of this study. The findings showed that indeed exist a statistically significant correlation between cyberbullying via cell phone or internet and the strati 1 to 5 ; in the same way showed that exist statistically significant correlations between being male and being cybervictim in the internet. There is also a direct relationship between being male and being cyberbully via internet or cell phone. The discussion also shows the importance of a further investigation in those variables, especially in the socioeconomic division since that kind of division is popular in developing countries.
\end{abstract}

Keywords:

Cyberbullying, factors of risk, gender, socioeconomic stratum, features of personality.

Bitácora del Artículo:

| Recibido: 1 de Febrero de 2018 | Aceptado: 1 Abril de 2018 | Publicado en línea: Enero-Junio de 2018 | 


\title{
Autoría y Derechos de Propiedad Intelectual
}

\section{FACTORES PERSONALES, DE GÉNERO Y SOCIOECONÓMICOS \\ RELACIONADOS CON EL CYBERBULLYING EN ADOLESCENTES \\ COLOMBIANOS}

\author{
William Fernando Morales Portilla, Ángela Yamile Rueda Forero, Jesús Redundo Pacheco, Marianela \\ Luzardo y Nataly Gómez Correa \\ Universidad Pontificia Bolivariana \\ Colombia
}

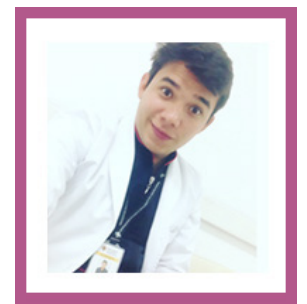

William Fernando Morales Portilla Universidad Pontificia Bolivariana Correo: william.Morales.2013@upb.edu.co

Psicólogo de la Facultad de Ciencias Sociales de la Universidad Pontíficia Bolivariana, Colombia.

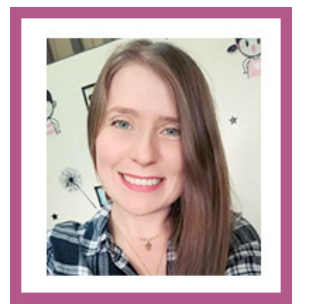

Ángela Yamile Rueda Forero Universidad Pontificia Bolivariana Correo: angela.Rueda.2013@upb.edu.co

Mujer emprendedora y perseverante, con gran capacidad de trabajo en equipo y liderazgo, que se rige con principios y valores, con un gran compromiso social y actitud investigadora.

Ver más...

\section{CONTRIBUCIÓN DE LOS AUtORES}

William Morales coordinó y contribuyó en todo el proceso metodológico y conceptual. | Ángela Rueda contribuyó en el proceso conceptual y metodológico. | Jesús Redundo revisó cada una de las partes del proyecto. | Marianela Luzardo realizó el análisis en el programa SPSS. | Nataly Gómez realizó parte de las conclusiones.

\section{AGRADECIMIENTOS}

[Agradecimiento por parte de los autores a intituciones, personas, etc. participantes en el estudio.]

\section{DAtos de Filiación de los Autores}

Universidad Pontificia Bolivariana, Bucaramanga, Colombia

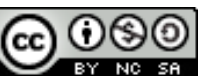

Copyright: (c) 2018 Morales Portilla, W. F., Rueda Forero, A. Y., Redundo Pacheco, J., Luzardo, M., \& Gómez Correa, N.

Este es un artículo de acceso abierto distribuido bajo los términos de la licencia Creative Commons Reconocimiento-NoComercial-Compartirlgual 4.0 Internacional, por lo que su contenido gráfico y escrito se puede compartir, copiar y redistribuir total o parcialmente sin necesidad de permiso expreso de sus autores con la única condición de que no se puede usar con fines directamente comerciales y los términos legales de cualquier trabajo derivado deben ser los mismos que se expresan en la presente declaración. La única condición es que se cite la fuente con referencia a la Revista Digital Internacional de Psicología y Ciencia Social y a sus autores. 


\section{TABLA DE CONTENIDO}

\begin{tabular}{lr} 
INTRODUCCIÓN & 43 \\
\hline \hline MÉTODO & 44 \\
\hline \hline \multicolumn{1}{l}{ Participantes, 44} & \\
$\quad$ Materiales, 44 & \\
$\quad$ Procedimiento, 44 & \\
$\quad$ Mediciones, 44 & \\
$\quad$ Análisis estadístico, 44 & 45 \\
RESULTADOS & 47 \\
\hline \hline DISCUSIÓN & 49 \\
\hline \hline & \\
\hline \hline
\end{tabular}




\section{INTRODUCCIÓN}

$\mathrm{E}$ I cyberbullying se define como una forma de intimidación entre iguales que se ejerce mediante dispositivos electrónicos para hostigar y acosar a los compañeros (Arab \& Díaz, 2015; Buelga \& Pons, 2012; Garaigordobil, 2011; Garaigordobil, Martínez-Valderrey \& Machimbarre, en prensa; García-Maldonado, Joffer-Velázquez, Martínez-Salazar \& Llanes-Castillo, 2011; Whittaker \& Kowalski, 2015). De esta manera, es importante determinar las variables implicadas que pueden influir en la aparición, desarrollo y mantenimiento del ciberacoso como edad, género, nivel socioeconómico y algunos rasgos de personalidad, entre otros.

Algunos autores afirman que las conductas relacionadas con el ciberostigamiento presentan un incremento en adolescentes con un promedio de edad de 14 años; a los 15 años hay una disminución del fenómeno, y de los 16 a los 18 años se mantienen o aumentan las conductas, así como también se ha evidenciado que los alumnos de 15 y 16 años son más víctimas que los alumnos de 12 y 13 años por medio del teléfono celular e internet (Del Barco, Mira, Verdasca, Castaño \& Gómez, 2013; Garaigordobil, 2015; Ortega-Baron, Buelga \& Cava, 2016). Por el contrario, otros estudios encontraron una relación positiva con la victimización y mayor incidencia alrededor de los 13 a los 15 años (Garaigordobil, 2011; García, 2013; Tokunaga, 2010). Asimismo, otras investigaciones indican que existe mayor cibervictimización en los grados séptimo y octavo, y después se produce un descenso de la misma (Schneider, O'DonneII, Stueve \& Coulter, 2012; Tokunaga, 2010).

De igual modo, el ciberacoso se relaciona directamente con el género, destacando estudios que afirman que esta forma de acoso se da en mayor porcentaje en mujeres que en hombres, ya sea como víctimas o como victimarias (Buelga, Cava \& Musitu, 2010; Estévez, Villardón, Calvete, Padilla \& Orue, 2010; Giménez, Maquilón \& Arnaiz, 2015; Sabater \& López-Hernáez, 2015). De esta manera, estudios señalan que las mujeres tienden a ser en mayor porcentaje víctimas, mientras que los hombres en mayor porcentaje victimarios (Del Barco, Mira, Verdasca, Castaño \& Gómez, 2013; Varela, Pérez, Schwaderer, Astillo \& Lecannelier, 2010).
Otra de las variables que se relaciona con el ciberacoso es el nivel socioeconómico, que es entendido en Colombia como el mecanismo que permite clasificar a la población en distintos estratos o grupos de personas que tienen características sociales y económicas similares, se dividen de uno hasta seis, siendo uno el más bajo y el seis el más alto, dependiendo de la heterogeneidad económica y social de las viviendas (Secretaría Distrital de Planeación, 2015). De esta manera, se evidencia en diferentes estudios que el cyberbullying se presenta con mayor frecuencia en estratos socioeconómicos altos, existiendo una relación directa entre el nivel socioeconómico alto y la ciberagresión, así como nivel socioeconómico bajo y la cibervictimización (Guerrero, Moncayo \& Parra, 2015; Santoyo \& Frías, 2014). Sin embargo, Erdur-Baker y Kavşut (2007) indican que no existe una relación significativa entre el cyberbullying y el nivel socioeconómico porque la prevalencia entre victimización y perpetración es semejante en los sujetos de nivel socioeconómico bajo y medio.

Por otra parte, la violencia, al igual que muchas conductas, es el reflejo de la personalidad, de aquello en lo que se cree, se valora y se defiende (Batrina, 2014; García, 2013; Hernández \& Solano, 2007; Jiménez-Bautista, 2012). De esta manera, existen determinados rasgos de personalidad que pueden hacer vulnerable a una persona en esta dinámica violenta; por ejemplo, se ha encontrado que el ciberagresor puede presentar conductas como baja satisfacción por la vida, baja autoestima, conductas antisociales y violentas, y bajo grado de autocontrol, entre otras (Buelga, Irazo, Cava \& Torralba, 2015; Garaigordobil, 2016; Povedano, Estévez, Martínez \& Monreal, 2012).

Asimismo, otros factores que se relacionan con este fenómeno son: percibir que el cyberbullying no tiene consecuencias graves, falta de apoyo entre iguales y considerar que en internet no hay normas (González, 2016; Sabater \& López-Hernáez, 2015). De igual modo, se han asociado otros factores de riesgo al ciberacosado, como problemas emocionales, baja autoestima, altos niveles de ansiedad, pocas habilidades sociales y conductas antisociales, entre otras (García-Maldonado et al., 2012; Ministère d'Éducation Nationale, 2011; Planas, 2013).

En este sentido, la presente investigación busca relacionar algunas variables que pueden influir como un factor de riesgo en este fenómeno, porque si se conocen las causas se pueden elaborar programas de prevención e intervención que permitan reducir los índices del cyberbullying. 


\section{MÉTOdO}

\section{Participantes}

La muestra estuvo constituida por 404 estudiantes de secundaria de dos centros educativos de Bucaramanga, Santander (58\% de una institución pública y $42 \%$ de una privada), con edades comprendidas entre los 10 y 19 años $(M=12.9$; DT $=1.89)$ de los que $49.5 \%$ son varones y $50.5 \%$ mujeres. El muestreo fue no probabilístico por conveniencia, porque no se hizo de forma aleatoria, dado que se seleccionó la población con base en las características similares a la muestra objeto. En la tabla 1 se presenta la distribución de la muestra por género y grado.

Tabla 1.

Número (y porcentaje) de sujetos de la muestra clasificados por género y curso.

\begin{tabular}{|l|c|c|c|}
\multirow{4}{*}{ Grado } & \multicolumn{3}{c}{ Género } \\
\hline \multirow{3}{*}{ Sexto } & 44 & 31 & 75 \\
\cline { 2 - 4 } & $(10.9 \%)$ & $(7.7 \%)$ & $(18.6 \%)$ \\
\hline \multirow{2}{*}{ Séptimo } & 33 & 35 & 68 \\
\cline { 2 - 4 } & $(8.2 \%)$ & $(8.7 \%)$ & $(16.8 \%)$ \\
\hline \multirow{2}{*}{ Octavo } & 30 & 35 & 65 \\
\cline { 2 - 4 } & $(7.4 \%)$ & $(8.7 \%)$ & $(16.1 \%)$ \\
\hline \multirow{3}{*}{ Noveno } & 33 & 35 & 68 \\
\cline { 2 - 4 } & $(8.2 \%)$ & $(8.7 \%)$ & $(16.8 \%)$ \\
\hline \multirow{2}{*}{ Décimo } & 29 & 39 & 68 \\
\cline { 2 - 4 } & $(7.2 \%)$ & $(9.7 \%)$ & $(16.8 \%)$ \\
\hline \multirow{2}{*}{ Undécimo } & 31 & 29 & 60 \\
\cline { 2 - 4 } & $(7.7 \%)$ & $(7.2 \%)$ & $(14.9 \%)$ \\
\hline \multirow{2}{*}{ Total } & 200 & 204 & 404 \\
\cline { 2 - 4 } & $(49.5 \%)$ & $(50.5 \%)$ & $(100 \%)$ \\
\hline
\end{tabular}

Por medio de la prueba chi-cuadrado de independencia entre las variables se comprueba que no existe relación estadísticamente significativa entre las variables grado y género $\left(x^{2}=4.254 ; p=0.514\right)$.

\section{Materiales}

PPG-IPG (Gordon, 1993). Este instrumento está basado en la unión de dos escalas: el perfil personal (PPG) y el inventario personal (IPG). Las dos pruebas presentan mediciones que integran la personalidad; por consiguiente, su incorporación en un solo instrumento permite la ob- tención de información de gran utilidad. Pretende medir rasgos de la personalidad, además de un factor de autoestima que se relaciona con la adaptación del sujeto en situaciones laborales, educativas y sociales. Consta de 38 ítems y evalúa nueve rasgos de personalidad: ascendencia, responsabilidad, estabilidad emocional, sociabilidad, autoestima, comprensión en las relaciones personales, originalidad, y vigor y cautela. En nuestro estudio el instrumento tuvo con una consistencia interna de 0.72.

Cuestionario Cyberbullying (Ortega, Calmaestra \& Mora Merchán, 2007, citados en Ortega, Calmaestra \& Mora Merchán, 2008). Este cuestionario explora las conductas de los jóvenes con el uso de teléfonos celulares e internet en relación con el cyberbullying. Está compuesto por 27 ítems, 10 de clasificación y 17 respecto a experiencias escolares acerca del bullying y del cyberbullying; además se incluye un apartado concreto acerca de los efectos del cyberbullying y las estrategias de afrontamiento con las que cuentan las víctimas respecto al mismo. En este caso, el cuestionario tiene una consistencia interna de 0.72 .

\section{Procedimiento}

Se realizó la gestión y aprobación en cada una de las instituciones que iban a participar en la investigación. Después de obtener el aval por parte de los directivos se informó acerca de los objetivos de la investigación. En seguida se hicieron los respectivos cronogramas de aplicación de los instrumentos (PPG-IPG y Cuestionario de Cyberbullying) acompañados de un cuestionario sociodemográfico. El protocolo del consentimiento informó de los objetivos del estudio, el carácter de voluntariedad, la protección de datos personales y garantías de confidencialidad, el derecho a la información y la posibilidad de abandonar el estudio en cualquiera de sus fases. La aplicación se hizo de manera anónima y confidencial, en donde cada participante tuvo un tiempo máximo de una hora; una vez conformada la base de datos, se analizaron con el paquete estadístico SPSS (versión 23).

\section{Mediciones}

Se realiza la corrélación entre las siguientes variables

1. Estrato socioeconómico y cyberbullying

2. Edad y cyberbullying

3. Género y cyberbullying

4. Caracteristicas de personalidad y cyberbullying

5. Agresor y cyberbullying

\section{Análisis estadístico}

El analisis se hizo por medio del paquete estadistico SPSS. Se utilizaron las pruebas chi-cuadrado para Co- 
nocer la relación entre el género y el grado que cursan los estudiantes (tabla 1). Por otro lado, para conocer el riesgo de agresión y victimización por teléfono celular e internet y el estrato social del individuo, se realizó una regresión logística teniendo como variables dependientes el hecho de ser o no cibervíctima o ciberagresor, y como variable independiente el estrato social, tomando como referencia el estrato 5 . De igual manera, se procedió para conocer el riesgo de estas variables y la edad, tomando como referencia al género femenino.

\section{Resultados}

Los resultados demostraron que existen relaciones estadísticamente significativas entre el ciberacoso efectuado por medio del teléfono celular o de internet y los estratos del 2 al 5, a excepción del estrato 1, correlacionado con la cibervictimización por teléfono celular, en donde no se halló relación (tabla 2). Sin embargo, se encontró que el riesgo de ser víctima por medio del teléfono celular respecto al estrato 5 disminuye en $51.1 \%$ con el estrato 2 , en $56.6 \%$ con el estrato 3 , así como en $4 \%$ con el estrato 4. En cuanto al riesgo de ser víctima por internet, los datos evidencian una disminución del riesgo en $36.6 \%$ entre el pertenecer al estrato 2 respecto al estrato 5 .

Respecto a la ciberagresion por medio del teléfono celular, según el estrato, se encontró que —en relación con el estrato 5 - pertenecer al estrato 1 disminuye el riesgo en $83.3 \%$, en $63.3 \%$ al estrato 2 , en $52.3 \%$ al estrato 3 y en $62.1 \%$ al estrato 4 . Por otra parte, los datos evidencian que el riesgo de ser agresor por internet disminuye, frente al estrato 5 , en $83.3 \%$ ser de estrato 1 , en $51.1 \%$ ser de estrato 2 , en $65.5 \%$ ser de estrato 3 y en $72.3 \%$ ser de estrato 4 .

Tabla 2.

Relación entre la variable estrato socioeconómico y la variable cyberbullying.

\begin{tabular}{|c|c|c|c|c|}
\hline \multirow{2}{*}{ ESTRATO } & \multicolumn{2}{|c|}{ CiBervíctima } & \multicolumn{2}{|c|}{ Ciberagresor } \\
\hline & Celular & INTERNET & Celular & INTERNET \\
\hline 1 & 0.750 & $1.000^{*}$ & $0.167^{*}$ & $0.167^{*}$ \\
\hline 2 & $0.489 *$ & $0.634^{* *}$ & $0.367^{*}$ & $0.489 *$ \\
\hline 3 & $0.434^{*}$ & $0.857^{* *}$ & $0.477^{*}$ & $0.345^{*}$ \\
\hline 4 & $0.600 * *$ & $1.034^{*}$ & $0.379^{*}$ & $0.277^{*}$ \\
\hline \multicolumn{5}{|c|}{ * Significativa al $1 \%}$. \\
\hline \multicolumn{5}{|c|}{ ** Significativa al 5\%. } \\
\hline *** Signific & a al $10 \%$. & & & \\
\hline
\end{tabular}

Respecto a la relación de la variable de edad y la condición de ser cibervíctima o ciberagresor, tanto por medio del teléfono celular como por internet, se encontró que sólo existe relación estadísticamente significativa a un nivel de significación de $10 \%$ entre estas dos variables. Además, se aprecia que dicha correlación es débil e inversa $(p=0.083<0.10)$ (tabla 3$)$.

Tabla 3.

Correlación entre la variable edad y la variable de cyberbuIlying.

\begin{tabular}{|c|c|c|c|c|c|c|}
\hline \multirow{2}{*}{ EdAD } & \multicolumn{6}{|c|}{ Cibervíctima } \\
\hline & Cel & $\times 2$ & $\mathbf{P}$ & INTR & $\times 2$ & $\mathbf{P}$ \\
\hline \multirow{2}{*}{$10-11$} & 35 & \multirow{10}{*}{12.5} & \multirow{10}{*}{0.354} & 46 & \multirow{10}{*}{0.14} & \multirow{10}{*}{0.354} \\
\hline & $8.7 \%$ & & & $11.4 \%$ & & \\
\hline \multirow{2}{*}{$12-13$} & 23 & & & 38 & & \\
\hline & $5.7 \%$ & & & $9.4 \%$ & & \\
\hline \multirow{2}{*}{$14-15$} & 48 & & & 41 & & \\
\hline & $11.9 \%$ & & & $10.1 \%$ & & \\
\hline \multirow{2}{*}{$15-16$} & 12 & & & 9 & & \\
\hline & $3 \%$ & & & $2.2 \%$ & & \\
\hline \multirow{2}{*}{$17-18$} & 1 & & & 0 & & \\
\hline & $2 \%$ & & & $0 \%$ & & \\
\hline \multirow{2}{*}{ EDAD } & \multicolumn{6}{|c|}{ Ciberagresor } \\
\hline & Cel & $\times 2$ & $\mathbf{P}$ & INTR & $\times 2$ & $\mathbf{P}$ \\
\hline \multirow{2}{*}{$10-11$} & 58 & \multirow{10}{*}{8.236} & \multirow{10}{*}{0.083} & 35 & \multirow{10}{*}{3.443} & \multirow{10}{*}{0.487} \\
\hline & $14.4 \%$ & & & $8.7 \%$ & & \\
\hline \multirow{2}{*}{$12-13$} & 57 & & & 28 & & \\
\hline & $14.1 \%$ & & & $6.9 \%$ & & \\
\hline \multirow{2}{*}{$14-15$} & 54 & & & 32 & & \\
\hline & $12.4 \%$ & & & $7.9 \%$ & & \\
\hline \multirow{2}{*}{$15-16$} & 15 & & & 8 & & \\
\hline & $3.7 \%$ & & & $2.0 \%$ & & \\
\hline \multirow{2}{*}{$17-18$} & 2 & & & 0 & & \\
\hline & $5 \%$ & & & $0 \%$ & & \\
\hline
\end{tabular}

Además los resultados mostraron relaciones estadísticamente significativas entre el género masculino y ser cibervíctima por internet. De igual modo existe una relación directa entre el género masculino y ser ciberagresor por medio de internet y el teléfono celular (tabla 4). En 
este sentido, el riesgo de ser cibervíctima por medio del teléfono celular disminuye en $17 \%$ respecto al género masculino frente al género femenino. Asimismo se puede evidenciar que el riesgo de ser ciberagresor por medio del teléfono celular disminuye en $56 \%$ al pertenecer al género masculino frente al femenino. A su vez, ser un estudiante de género masculino disminuye $61 \%$ respecto al riesgo de ser ciberagresor por medio del internet.

Tabla 4.

Correlación entre la variable género y la variable del cyberbullying.

\begin{tabular}{|c|c|c|c|c|}
\hline \multirow{2}{*}{ GÉNERO } & \multicolumn{2}{|c|}{ Cibervíctima } & \multicolumn{2}{|c|}{ Ciberagresor } \\
\hline & Celular & INTERNET & Celular & INTERNET \\
\hline Masculino & 0.826 & $0.531^{*}$ & $0.432^{*}$ & $0.382^{*}$ \\
\hline \multicolumn{5}{|l|}{ Femenino } \\
\hline \multicolumn{5}{|c|}{ * Significativa al $1 \%$. } \\
\hline \multicolumn{5}{|c|}{ ** Significativa al 5\%. } \\
\hline$* * *$ Significativa & & & & \\
\hline
\end{tabular}

Tabla 5.

Correlación entre la variable rasgos de personalidad y la variable del cyberbullying.

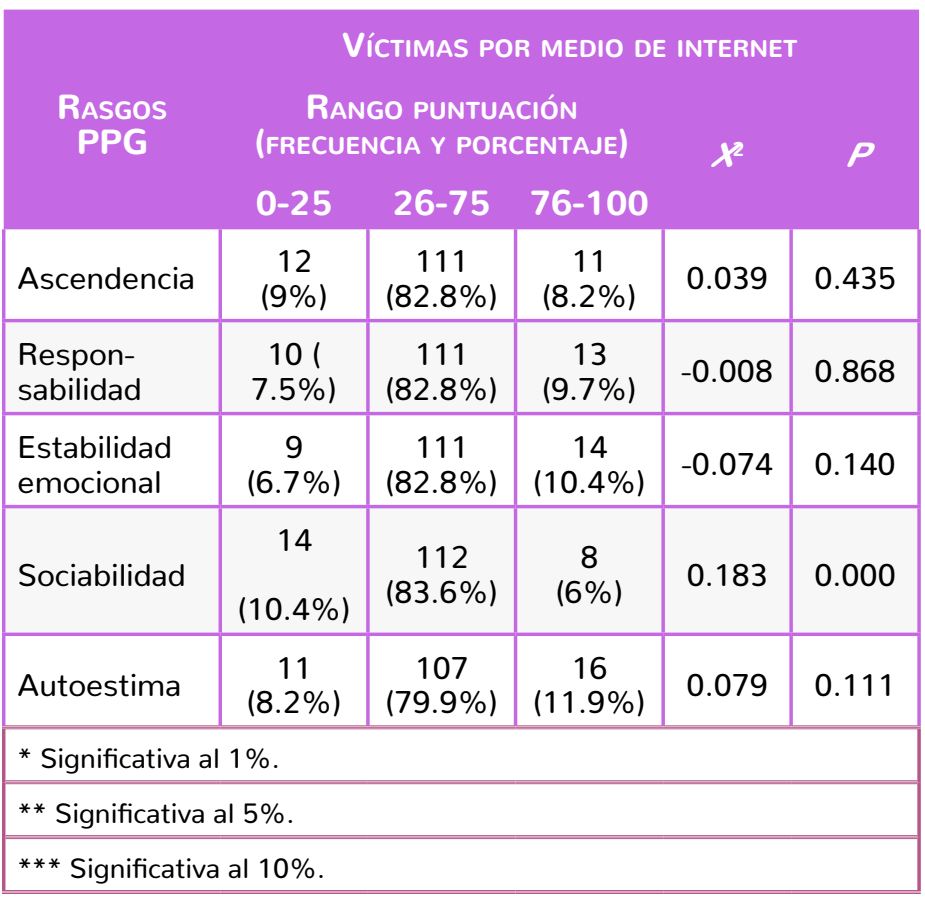

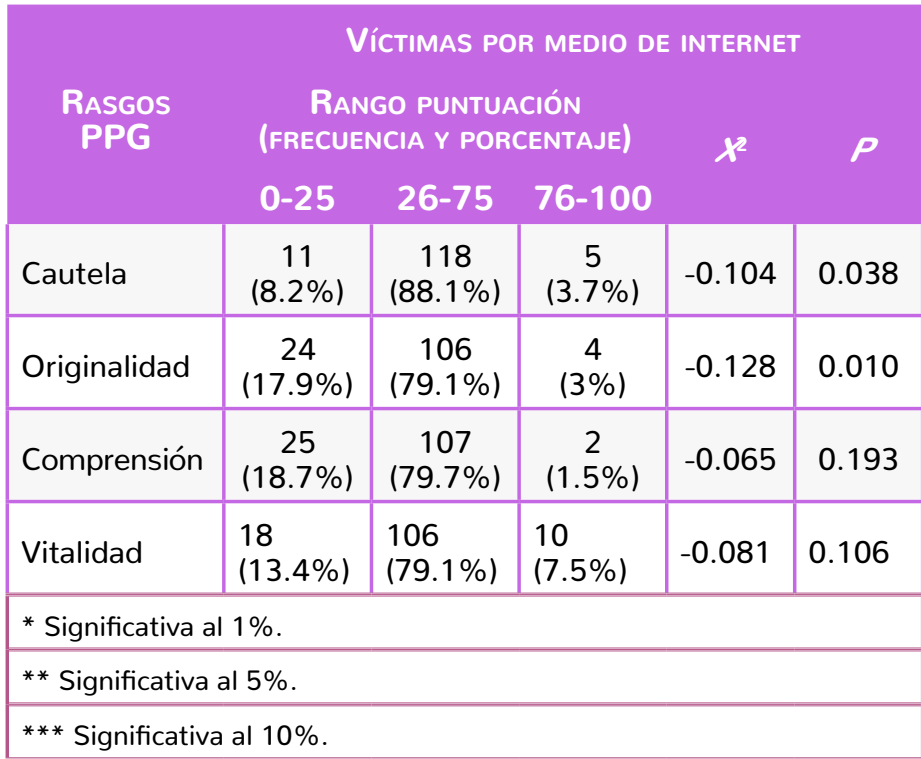

\begin{tabular}{|c|c|c|c|c|c|}
\hline \multirow[t]{2}{*}{$\begin{array}{l}\text { RASGOS } \\
\text { PPG }\end{array}$} & \multicolumn{3}{|c|}{$\begin{array}{l}\text { RANGO PUNTUACIÓN } \\
\text { (FRECUENCIA Y PORCENTAJE) }\end{array}$} & \multirow{2}{*}{$x^{2}$} & \multirow[t]{2}{*}{$\boldsymbol{P}$} \\
\hline & $0-25$ & $26-75$ & $76-100$ & & \\
\hline Ascendencia & $\begin{array}{c}22 \\
(11.8 \%)\end{array}$ & $\begin{array}{c}151 \\
(81.2 \%)\end{array}$ & $\begin{array}{c}13 \\
(7 \%)\end{array}$ & -0.034 & 0.502 \\
\hline $\begin{array}{l}\text { Respon- } \\
\text { sabilidad }\end{array}$ & $\begin{array}{c}14 \\
(7.5 \%)\end{array}$ & $\begin{array}{c}156 \\
(83.9 \%)\end{array}$ & $\begin{array}{c}16 \\
(8.6 \%)\end{array}$ & -0.037 & 0.459 \\
\hline $\begin{array}{l}\text { Estabilidad } \\
\text { emocional }\end{array}$ & $\begin{array}{c}14 \\
(7.5 \%)\end{array}$ & $\begin{array}{c}151 \\
(81.2 \%)\end{array}$ & $\begin{array}{c}21 \\
(11.3 \%)\end{array}$ & -0.096 & 0.054 \\
\hline Sociabilidad & $\begin{array}{c}30 \\
(16.1 \%)\end{array}$ & $\begin{array}{c}145 \\
(78 \%)\end{array}$ & $\begin{array}{c}11 \\
(5.9 \%)\end{array}$ & 0.122 & 0.015 \\
\hline Autoestima & $\begin{array}{c}21 \\
(11.3 \%)\end{array}$ & $\begin{array}{c}147 \\
(79 \%)\end{array}$ & $\begin{array}{c}18 \\
(9.7 \%)\end{array}$ & 0.002 & 0.964 \\
\hline Cautela & $\begin{array}{c}13 \\
(7 \%)\end{array}$ & $\begin{array}{c}166 \\
(89.2 \%)\end{array}$ & $\begin{array}{c}7 \\
(3.8 \%)\end{array}$ & -0.103 & 0.038 \\
\hline Originalidad & $\begin{array}{c}32 \\
(17.2 \%)\end{array}$ & $\begin{array}{c}144 \\
(77.4 \%)\end{array}$ & $\begin{array}{c}10 \\
(5.4 \%)\end{array}$ & -0.101 & 0.042 \\
\hline Comprensión & $\begin{array}{c}33 \\
(17.7 \%)\end{array}$ & $\begin{array}{c}149 \\
(80.1 \%)\end{array}$ & $\begin{array}{c}4( \\
2.2 \%) \\
\end{array}$ & -0.050 & 0.318 \\
\hline Vitalidad & $\begin{array}{c}25 \\
113.4 \% \\
\end{array}$ & $\begin{array}{c}144 \\
(77.4 \%) \\
\end{array}$ & $\begin{array}{c}17 \\
(9.1 \%) \\
\end{array}$ & -0.075 & 0.133 \\
\hline \multicolumn{6}{|c|}{ * Significativa al $1 \%$. } \\
\hline \multicolumn{6}{|c|}{ ** Significativa al 5\%. } \\
\hline \multicolumn{6}{|c|}{ *** Significativa al $10 \%}$. \\
\hline
\end{tabular}


Tabla 6.

Correlación entre la variable rasgos de personalidad y la variable agresores del cyberbullying.

\begin{tabular}{|c|c|c|c|c|c|}
\hline \multirow{3}{*}{$\begin{array}{l}\text { RASGOS } \\
\text { PPG }\end{array}$} & \multicolumn{5}{|c|}{ AGRESOR POR MEDIO DE INTERNET } \\
\hline & \multicolumn{3}{|c|}{$\begin{array}{c}\text { RANGO PUNTUACIÓN } \\
\text { (FRECUENCIA Y PORCENTAJE) }\end{array}$} & \multirow{2}{*}{$x^{2}$} & \multirow{2}{*}{$\boldsymbol{P}$} \\
\hline & $0-25$ & $26-75$ & $76-100$ & & \\
\hline Ascendencia & $\begin{array}{c}10 \\
(9.7 \%)\end{array}$ & $\begin{array}{c}83 \\
(80.6 \%)\end{array}$ & $\begin{array}{c}10 \\
(9.7 \%)\end{array}$ & 0.042 & 0.398 \\
\hline $\begin{array}{l}\text { Respon- } \\
\text { sabilidad }\end{array}$ & $\begin{array}{c}6 \\
(5.8 \%)\end{array}$ & $\begin{array}{c}86 \\
(83.5 \%)\end{array}$ & $\begin{array}{c}11 \\
(10.7 \%)\end{array}$ & 0.030 & 0.544 \\
\hline $\begin{array}{l}\text { Estabilidad } \\
\text { emocional }\end{array}$ & $\begin{array}{c}8 \\
(7.8 \%)\end{array}$ & $\begin{array}{c}84 \\
(81.6 \%)\end{array}$ & $\begin{array}{c}11 \\
(10.7 \%)\end{array}$ & -0.072 & 0.150 \\
\hline Sociabilidad & $\begin{array}{c}12 \\
(11.7 \%)\end{array}$ & $\begin{array}{c}85 \\
(82.5 \%)\end{array}$ & $\begin{array}{c}6 \\
(5.8 \%)\end{array}$ & 0.134 & 0.007 \\
\hline Autoestima & $\begin{array}{c}13 \\
(12.6 \%)\end{array}$ & $\begin{array}{c}80 \\
(77.7 \%)\end{array}$ & $\begin{array}{c}10 \\
(9.7 \%)\end{array}$ & -0.014 & 0.775 \\
\hline Cautela & $\begin{array}{c}9 \\
(8.7 \%)\end{array}$ & $\begin{array}{c}90 \\
(87.4 \%)\end{array}$ & $\begin{array}{c}4 \\
(3.9 \%)\end{array}$ & -0.092 & 0.064 \\
\hline Originalidad & $\begin{array}{c}20 \\
(19.4 \%)\end{array}$ & $\begin{array}{c}80 \\
(77.7 \%)\end{array}$ & $\begin{array}{c}3 \\
(2.9 \%)\end{array}$ & -0.128 & 0.010 \\
\hline Comprensión & $\begin{array}{c}17 \\
(16.5 \%)\end{array}$ & $\begin{array}{c}83 \\
(80.6 \%)\end{array}$ & $\begin{array}{c}3 \\
(2.9 \%)\end{array}$ & -0.003 & 0.949 \\
\hline Vitalidad & $\begin{array}{c}14 \\
(13.6 \%)\end{array}$ & $\begin{array}{c}84 \\
(81.6 \%)\end{array}$ & $\begin{array}{c}5 \\
(4.9 \%)\end{array}$ & -0.099 & 0.046 \\
\hline \multicolumn{6}{|c|}{ * Significativa al $1 \%$. } \\
\hline \multicolumn{6}{|c|}{ ** Significativa al 5\%. } \\
\hline ** Significat & & & & & \\
\hline
\end{tabular}

\begin{tabular}{|c|c|c|c|c|c|}
\hline \multirow{4}{*}{$\begin{array}{l}\text { RASGOS } \\
\text { PPG }\end{array}$} & \multicolumn{5}{|c|}{ Agresor por MEdio del teléFono CELULAR } \\
\hline & \multirow{2}{*}{\multicolumn{3}{|c|}{$\begin{array}{l}\text { RANGO PUNTUACIÓN } \\
\text { (FRECUENCIA Y PORCENTAJE) }\end{array}$}} & \multirow{3}{*}{$x^{2}$} & \multirow{3}{*}{$\boldsymbol{P}$} \\
\hline & & & & & \\
\hline & $0-25$ & $26-75$ & $76-100$ & & \\
\hline Ascendencia & $\begin{array}{c}12 \\
(10.1 \%)\end{array}$ & $\begin{array}{c}96 \\
(80.7 \%)\end{array}$ & $\begin{array}{c}11 \\
(9.2 \%)\end{array}$ & 0.034 & 0.491 \\
\hline $\begin{array}{l}\text { Respon- } \\
\text { sabilidad }\end{array}$ & $\begin{array}{c}9 \\
(7.6 \%)\end{array}$ & $\begin{array}{c}101 \\
(84.9 \%)\end{array}$ & $\begin{array}{c}9 \\
(7.6 \%)\end{array}$ & -0.043 & 0.392 \\
\hline $\begin{array}{l}\text { Estabilidad } \\
\text { emocional }\end{array}$ & $\begin{array}{c}8 \\
(6.7 \%)\end{array}$ & $\begin{array}{c}95 \\
(79.8 \%)\end{array}$ & $\begin{array}{c}16 \\
(13.4 \%)\end{array}$ & -0.024 & 0.625 \\
\hline Sociabilidad & $\begin{array}{c}19 \\
(16 \%)\end{array}$ & $\begin{array}{c}93 \\
(78.2 \%)\end{array}$ & $\begin{array}{c}7 \\
(5.9 \%)\end{array}$ & 0.087 & 0.081 \\
\hline \multicolumn{6}{|c|}{ * Significativa al $1 \%$. } \\
\hline \multicolumn{6}{|c|}{ ** Significativa al $5 \%$. } \\
\hline \multicolumn{6}{|c|}{ *** Significativa al $10 \%$. } \\
\hline
\end{tabular}

\begin{tabular}{|c|c|c|c|c|c|}
\hline \multirow{3}{*}{$\begin{array}{l}\text { RASGOS } \\
\text { PPG }\end{array}$} & \multicolumn{5}{|c|}{ AgRESOR POR MEDIO dEL TELÉFONO CELULAR } \\
\hline & \multicolumn{3}{|c|}{$\begin{array}{l}\text { RANGO PUNTUACIÓN } \\
\text { (FRECUENCIA Y PORCENTAJE) }\end{array}$} & \multirow{2}{*}{$x^{2}$} & \multirow{2}{*}{$\boldsymbol{P}$} \\
\hline & $0-25$ & $26-75$ & $76-100$ & & \\
\hline Autoestima & $\begin{array}{c}14 \\
(11.8 \%)\end{array}$ & $\begin{array}{c}91 \\
(76.5 \%)\end{array}$ & $\begin{array}{c}14 \\
(11.8 \%)\end{array}$ & 0.023 & 0.643 \\
\hline Cautela & $\begin{array}{c}11 \\
(9.2 \%)\end{array}$ & $\begin{array}{c}105 \\
(88.2 \%)\end{array}$ & $\begin{array}{c}3 \\
(2.5 \%)\end{array}$ & -0.136 & 0.006 \\
\hline Originalidad & $\begin{array}{c}23 \\
(19.3 \%)\end{array}$ & $\begin{array}{c}93 \\
(78.2 \%)\end{array}$ & $\begin{array}{c}3 \\
(2.5 \%)\end{array}$ & -0.146 & 0.003 \\
\hline Comprensión & $\begin{array}{c}20 \\
(16.8 \%)\end{array}$ & $\begin{array}{c}96 \\
(80.7 \%)\end{array}$ & $\begin{array}{l}3 \\
(2.5 \%)\end{array}$ & -0.014 & 0.773 \\
\hline Vitalidad & $\begin{array}{c}15 \\
(12.6 \%\end{array}$ & $\begin{array}{c}92 \\
(77.3 \%)\end{array}$ & $\begin{array}{c}12 \\
(10.1 \%)\end{array}$ & -0.029 & 0.566 \\
\hline \multicolumn{6}{|c|}{ * Significativa al $1 \%$. } \\
\hline \multicolumn{6}{|c|}{ ** Significativa al 5\%. } \\
\hline \multicolumn{6}{|c|}{$* * *$ Significativa al $10 \%$. } \\
\hline
\end{tabular}

\section{Discusión}

El objetivo principal de esta investigación fue identificar si el estrato socioeconómico, la edad, el género y la personalidad son factores de riesgo en el cyberbullying. Así, respecto al estrato socioeconómico algunos estudios se refieren a esta variable como un factor que puede influir en la manifestación de este fenómeno de violencia virtual, destacando la presencia de la misma en mayor proporción en estratos altos $(4,5,6)$ que en estratos bajos $(1,2,3)$, lo que se podría explicar debido al mayor acceso a los medios virtuales en los estratos altos, aunque la conclusión fue que no presentaban una amplia diferencia (Guerrero, Moncayo \& Parra, 2015; Santoyo \& Frías, 2014). Ahora bien, los resultados de nuestra investigación mostraron que en cualquiera de los cinco estratos estudiados se puede presentar el fenómeno del cyberbuIlying, producto similar al obtenido por Garaigordobil, Aliri, Maganto, Bernarás \& Jaureguiza (2014) en España, donde se demostró que la relación entre la cibervictimización-ciberagresión, y las conductas de cyberbullying sufridas fue similar en los distintos estratos socioeconómicos. Asimismo, en un estudio de Menay-López \& Fuente-Mella (2014) en Chile, donde encontraron que no hay diferencias significativas en la presentación de este fenómeno respecto a los estratos socioeconómicos. Por último, no existen suficientes investigaciones en Colombia, y las existentes no muestran resultados similares a los de Guerrero, Moncayo \& Parra (2015). Sin embargo, en 
nuestra investigación se evidencia que la estratificación no se identificaría como un factor de riesgo porque en cualquier estrato se podría presentar ciberbullying, tal vez porque la distribución de estratos o zonas económicas sólo existe en países en vía de desarrollo, sumado a que la investigación general acerca del ciberbullying apenas comienza en nuestro país.

En cuanto a la variable género, se encontró que ser hombre es un factor de riesgo para ser víctima por medio del teléfono celular, además de ser victimario, tanto por internet como mediante el teléfono celular, al contrario de los resultados obtenidos en diversas investigaciones donde se destaca que ser mujer incide más en la presencia de este fenómeno, ya sea como víctimas o como victimarias, ya que las mujeres prefieren acosar a sus pares por medio de las nuevas tecnologías, donde no interesa estar en desventaja física, llevando a consecuencias más crónicas y a la propagación y perpetuación del fenómeno, contrario a los hombres, porque éstos prefieren hacerlo de manera directa y ocasionar daños físicos (Buelga, Cava \& Musitu, 2010; Estévez, Villardón, Calvete, Padilla \& Orue, 2010; Giménez, Maquilón \& Arnaiz, 2015;Morales-Reynoso \& Serrano-Barquin, 2014; Sabater \& López-Hernáez, 2015).

Cosa parecida sucede con la correlación de las variables edad y ser cibervíctima o ciberagresor, debido a que varios autores afirman que éstas no se relacionan de modo directo, aunque se parte de la premisa que no existen estudios concluyentes frente a ésta (Slonge \& Smith, 2008). En un estudio hecho por Ortega, Calmaestra y Mora-Merchan (2008), en el que se tuvo como variable analizar la edad, no se encontró relación significativa entre ésta y ser cibervíctima o ciberagresor. De esta manera, el presente estudio mostró una relación de 10\%, lo que corrobora la baja relación de éstas.

Respecto a los rasgos de personalidad se evidenció una correlación significativa entre la cibervictimización y la sociabilidad, la cual obtuvo una puntuación alta. La sociabilidad hace referencia a individuos que les gusta estar y trabajar con otras personas en puntuaciones altas, y en puntuaciones bajas a personas que restringen sus contactos sociales; cautela que hace alusión en puntuaciones altas a individuos muy cuidadosos en la toma de decisiones, y en puntuaciones bajas a individuos precipitados, que corren riesgos; también presentó una correlación significativa en puntuaciones altas. Asimismo la originalidad fue relevante; ésta se refiere en puntuaciones altas a individuos que les gusta trabajar en problemas difíciles, reflexionan y piensan en nuevas ideas; en puntuaciones bajas, son personas que se caracterizan por el gusto a trabajar en problemas difíciles; por el contrario, cuando puntúa bajo se refiere a personas que no les gusta entrar en discusiones que obliguen a reflexionar (Rodríguez \& Garibay, 2014). Estos aspectos no presentaron diferencias en la modalidad de internet o celular. Sumado a esto, los rasgos significativos en el rol del ciberagresor fueron sociabilidad y originalidad asociado a la modalidad de internet; asimismo cautela y originalidad asociados a la modalidad de celular. Es importante recordar que la mayor frecuencia en todos los rasgos que resultan representativos se ubicó en la media, lo que significa que tener estos rasgos en la norma es un factor de riesgo tanto para ser víctima como para ser agresor.

Aunque estos resultados son relevantes, difieren de los encontrados en otras investigaciones, como las de Cerezo (2001), que afirma que los ciberagresores tienden a establecer dinámicas agresivas, tienen un autoconcepto muy elevado, son extrovertidos y sinceros, y tienen un alto nivel de psicoticismo y nivel medio de neuroticismo. Por lo contrario, las cibervíctimas suelen mostrarse tímidas, retraídas y aisladas socialmente; además se consideran poco sinceras y tienen un nivel alto de neuroticismo, ansiedad e intraversión. Al igual que el Ministère d'Éducation Nationale (2011), que habla de victimarios con visión agresiva, baja empatía y bajo reconocimiento del efecto de sus acciones y victimas con ansiedad, baja autoestima, anhedonia y trastornos del cueño. García-Maldonado et al. (2012) afirma en su investigación que rasgos de la personalidad como la hiperactividad son un factor de riesgo para el rol de cibervíctimas-ciberagresores, así como para el rol de cibervíctimas, los problemas emocionales influyen en la aparición del ciberbullying. Por consiguiente, los resultados de esta investigación reafirman la necesidad de continuar con los procesos investigativos del ciberbullying, porque la cobertura de las TIC ha aumentado en Colombia, al igual que en los países industrializados (Mura \& Diamantini, 2013). Además existe información escasa y limitada acerca de los factores de riesgo, y en general del fenómeno del ciberbullying, que obstaculizan los procesos de promoción, prevención e intervención pertinentes en Colombia. La presente investigación indica que los rasgos de personalidad y el género son un factor de riesgo del ciberbullying, y por tanto merecen la atención de todas las instituciones encargadas de regular dichas conductas violentas en la escuela; no obstante, el estrato socioeconómico es una variable no muy relevante, tal vez porque la presentación del ciberbullying no está condicionada a un espacio determinado. En conclusión, es importante que desde la psicología se trabaje con las características de la personalidad que este y diversos estudios han relacionado con el surgimiento y mantenimiento del ciberacoso; asimismo, es 
importante continuar con investigaciones descriptivas y experimentales en donde se logre establecer procesos de intervención considerando las variables reconocidas como factores de riesgo.

\section{Referencias}

Arab, E., \& Díaz, A. (2015). Impacto de las redes sociales e internet en la adolescencia: Aspectos positivos y negativos. Revista médica, 26 (1), 7-13. Recuperado de http://www.elsevier.es/es-revista-revista-medica-clinicalas-condes-202-articulo-impacto-las-redes-socialese-S0716864015000048.usitu

Barco, B. del, Mira, A. R., Verdasca, J. L., Castaño, E. P., \& Gómez, T. (2013). Cyberbullying en centros de enseñanza básica y secundaria del Alentejo (Portugal). Revista educacao, temas y problemas, 12, 239-251. Recuperado de https:// dspace.uevora.pt/rdpc/bitstream/10174/10669/4/Artigo Cyberbullying.pdf.

Batrina, M. J. (2014). Conductas de cyberacoso en niños y adolescentes: Hay una salida con la educación y la conciencia social. Educar, 50 (2), 383-400. Recuperado de http://ddd.uab.cat/pub/educar/educar a2014m712v50n2/educar a2014m7-12v50n2p383.pdf.

Buelga, S., Irazo, B., Cava, M., \& Torralba, E. (2015). Psychological profile of adolescent cyberbullying aggressors. International Journal of Social Psychology, 30 (2), 382406. Recuperado de https://www.researchgate.net/ publication/277351586 Psychological profile of adolescent cyberbullying aggressors Perfil psicosocial de_adolescentes_agresores_de_cyberbullying.

Buelga, S., Cava, M. J. \& Musitu, G. (2010). Cyberbullying: Victimización entre adolescentes a través del teléfono móvil y de Internet. Psicothema, 22, 784-789. Recuperado de http://www.psicothema.com/pdf/3802.pdf.

Buelga, S., \& Pons, J. (2012). Agresiones entre adolescentes a través del teléfono móvil y de internet. Copmadrid, 21 (1), 91-101. Recuperado de http://www.copmadrid.org/ webcopm/publicaciones/social/in2012v21n1a2.pdf.

Cerezo, F. (2001). Variables de personalidad asociadas en la dinámica bullying (agresores versus víctimas) en niños y niñas de 10 a 15 años. Anales de psicología, 17 (1), 3743. Recuperado de http://www.um.es/analesps/v17/ v17 1/04-17 1.pdf.

Erdur-Baker, Ö., \& Kavşut, F. (2007). Cyberbullying: a new face of peer bullying. Egitim Arastirmalari-Eurasian Journal of Educational Research, 27, 31-42. Recuperado de https:// www.researchgate.net/publication/284107943 Cyber bullying A new face of peer bullying.

Estévez, A., Villardón, L., Calvete, E., Padilla, P., \& Orue, I. (2010). Adolescentes víctimas de cyberbullying: Prevalencia y características. Revista de Psicología Clínica y de la Salud, 18, 73-89. Recuperado de http://www.funveca.org/ revista/pedidos/product.php?id product=373.

Garaigordobil, M. (2011). Prevalencia y consecuencias del cyberbullying: Una revisión. International Journal of Psychology and Psychological Therapy, 11 (2), 233-
254. Recuperado de http://www.redalyc.org/articulo. oa?id=56019292003.

Garaigordobil, M. (2015). Cyberbullying en adolescentes y jóvenes del País Vasco: Cambios con la edad. Anales de Psicología, 31 (3), 1069-1076. Recuperado de http://www. redalyc.org/pdf/167/16741429034.pdf.

Garaigordobil, M. (2016). Conducta antisocial: Conexión con bullying/cyberbullying y estrategias de resolución de conflictos. Psychosocial Intervention, 8. Recuperado de https://www.researchgate.net/ publication/291952167 Conducta antisocial_conexion con bullyingcyberbullying y estrategias de resolucion de conflictos.

Garaigordobil, M., Aliri, J., Maganto, C., Bernarás, E., \&Jaureguiza, J. (2014). Cyberbullying: Prevalencia de víctimas, agresores y observadores en función del nivel socio-económicocultural. Proceedings of International Congress of Clinical Psychology, 42-49. Recuperado de http://www.aepc.es/ PsClinicalX/PROCEEDING/7.pdf.

Garaigordobil, M., Martínez-Valderrey, V., \& Machimbarrena, J. (en prensa). Intervención en el bullying y Cyberbullying: Evaluación del caso Martín. Revista de Psicología Clínica con Niños y Adolescentes, 4 (1). Recuperado de http:// www.revistapcna.com/sites/default/files/15-18.pdf.

García, C. (2013). Acoso y ciberacoso en escolares de primaria: factores de personalidad y de contexto entre iguales (tesis doctoral). Córdoba: Universidad de Córdoba. Recuperado de http://helvia.uco.es/xmlui/bitstream/ handle/10396/10949/2013000000831.pdf?sequence=1.

García-Maldonado, G., Joffer-Velazquez, V. M., Martínez-Salazar, G. J., \& Llanes-Castillo, A. (2011). Cyberbullying: Forma virtual de intimidación escolar. Revista colombiana de psiquiatría, 40 (1), 115-130. Recuperado de http://www. scielo.org.co/pdf/rcp/v40n1/v40n1a10.pdf.

García-Maldonado, G., Martínez- Salazar, G. J., SaldívarGonzález, A., Sánchez-Nuncio, R., Martínez-Perales, G. M., \& Barrientos Gómez, M. (2012). Factores de riesgo y consecuencias del cyberbullying en un grupo de adolescentes: Asociación con bullying tradicional. Boletín médico del hospital infantil de México, 69 (6), 463-474. Recuperado de http://www.scielo.org.mx/pdf/bmim/ v69n6/v69n6a7.pdf.

Giménez, A. M., Maquilón, J. J., \& Arnaiz, P. (2015). Usos problemáticos y agresivos de las TIC por parte de adolescentes implicados en cyberbullying. Revista de Investigación Educativa, 33 (2), 335-351. DOI: http:// dx.doi.org/10.6018/rie.33.2.199841.

Gonzáles, A. (2016). Factores de riesgo en el ciberacoso: Revisión sistemática a partir del modelo del triple riesgo delictivo (TRD). Revista de internet, derecho y política, (22). Recuperado de journals.uoc.edu/index.php/idp/ article/view/n22-gonzalez.

Gordon, L. (1993). Gordon Personal Profile-Inventory. Recuperado de http://us.talentlens.com/wp-content/ uploads/pdf/Gordon\%20PP-I_Manual.pdf.

Guerrero, D., Moncayo, L., \& Parra, J. (2015). Cyberbullying El acoso escolar en la era virtual (trabajo de grado). Colombia: Instituto Merani. Recuperado de http:// www.institutomerani.edu.co/publicaciones/tesis/2015/ 
cyberbullying\%20 acoso escolar era\%20virtual.pdf.

Hernández, M., \& Solano, I. (2007). Cyberbullying, un problema de acoso escolar. Revista iberoamericana de educación a distancia, 10 (1), 17-36. Recuperado de http://www. biblioteca.org.ar/libros/141650.pdf.

Jiménez-Bautista, F. (2012). Conocer para comprender la violencia: Origen, causas y realidad. Convergencia, 58, 1352. Recuperado de http://www.scielo.org.mx/pdf/conver/ v19n58/v19n58a1.pdf.

Luengo, J. A. (2014). Guía de recursos didácticos para centros educativos. Madrid: Colegio Oficial de Psicólogos de Madrid. Recuperado de http://www.copmadrid.org/ webcopm/recursos/CiberbullyingB.pdf.

Menay-López, L., \& Fuente-Mella, H. (2014). Plataformas comunicacionales del ciberbullying. Una aplicación empírica en dos colegios de la quinta región, Chile. Estudios pedagógicos, 40, (2). Recuperado de http://www.scielo.cl/scielo.php?script=sci arttext\&pid=S0718-7052014000300007.

Ministère d'Éducation Nationale (2011). Guide pratique pour lutter contre le cyber-harcèlement entre élèves. Recuperado de http://media.education.gouv.fr/file/09 septembre/58/6/guide-cyberharcelement 190586.pdf.

Morales-Reynoso, T., \& Serrano-Barquin, T. (2014). Manifestaciones del cyberbullying por género entre los estudiantes de bachillerato. Revista Ra-Ximhai, 10 (2), 235-261. Recuperado de http://www.redalyc.org/ pdf/461/46132726010.pdf.

Mura, D., \& Diamantini, D. (2013). Cyberbullying among Colombian students: An exploratory investigation. European Journal of Investigation in Health, Psychology and Education, 3 (3), 249-256.

Ortega-Barón, J., Buelga, S., \& Cava, M. (2016). Influencia del clima escolar en adolescentes victimas de ciberacoso. Revista cientifica de educomunicación. 14(46). 57-65. Recuperado de www.revistacomunicar.com/verpdf. php?numero $=46 \&$ articulo $=46-2016-06$

Ortega, R., Calmaestra, J., \& Mora-Merchán, J. (2008). Ciberbullying. International Journal of Psychology and Psychological Therapy, 8 (2), 183-192. Recuperado de http://www.ijpsy.com/volumen8/num2/194/ cyberbullying-ES.pdf.

Planas, J. (2013). Cyberbullying: Una experiencia de prevención. Aula secundaria, 1, 10-13. Recuperado de https:// convivencia.files.wordpress.com/2013/03/ciberbull.pdf.

Povedano, A., Estévez, E., Martínez, B., \& Monreal, M. C. (2012). Un perfil psicosocial de adolescentes agresores y víctimas en la escuela: Análisis de las diferencias de género. Revista de Psicología Social, 27 (2), 169-182. Recuperado de https://www.uv.es/lisis/amapola/12-art1.pdf.
Rodríguez, C., \& Garibay, J. (2014). Análisis de rasgos de la personalidad profesional en estudiantes de licenciatura en Administración de una IES. Asamblea general de ALAFEC. Recuperado de http://www.alafec.unam.mx/ docs/asambleas/xiv/ponencias/10.03.pdf.

Sabater, C., \& López-Hernández, L. (2015). Factores de riesgo en el cyberbullying. Frecuencia y exposición de los datos personales en internet. International Journal of Sociology of Education, 4 (1), 1-25. Recuperado de http:// hipatiapress.info/hpjournals/index.php/rise/article/ view/1229/1126.

Santoyo, D., \& Frías, S. M. (2014). Acoso escolar en México: Actores involucrados y sus características. Revista latinoamericana de estudios educativos, 4, 13-41. Recuperado de http:// www.seg.guanajuato.gob.mx/Ceducativa/CDocumental/ Doctos/2015/Mar/02AcosoescolarMexico.pdf.

Schneider, S., O'Donnell, L., Stueve, A., \& Coulter, R. (2012). Cyberbullying, School Bullying, and Psychological Distress: A Regional Census of High School Students. American Journal of Public Health, 102 (1), 171-177. Recuperado de http://www.promoteprevent.org/sites/www. promoteprevent.org/files/resources/Cyberbullying\%20 school\%20bullying\%20and\%20psychological\%20distress. pdf.

Secretaría Distrital de Planeación (2015). Estratificación socioeconómica. Recuperado de http://www.sdp.gov. co/portal/page/portal/PortalSDP/InformacionTomaDec isiones/Estratificacion Socioeconomica/QueEs.

Slonge, R., y Smith, P. (2008). Cyberbullying: another main type of bullying. Scandinavian Journal of Psychology, 49, 147-154. Recuperado de https://www.ncbi.nlm.nih.gov/ pubmed/18352984.

Tokunaga, R. S. (2010). Following you home from school:uu A critical review and synthesis of research on cyberbullying victimization. Computers in Human Behavior, 26, 277-287. Recuperado de http://ac.els-cdn.com/ S074756320900185X/1-s2.0-S074756320900185X-main. pdf? tid=bb5dee46-8a3f-11e6-a76a-00000aab0f6b\&acd nat=1475591881 7481646048e8a4a2fc42ac68782007a2.

Varela, J., Pérez, J. C., Schwaderer, H., Astillo, J., \& Lecannelier, F. (2010). Caracterización del cyberbullying en el gran Santiago de Chile, en el año 2010. Revista Quadrimestral da Associação Brasileira de Psicologia Escolar e Educacional, 18 (2), 347-354. Recuperado de http://www.scielo.br/pdf/ pee/v18n2/1413-8557-pee-18-02-0347.pdf.

Whittaker, E., \& Kowalsky, R. (2015). Ciberbullying vía social media. Journal of school violence, 14 (1), 11-29. Recuperado de http://eric.ed.gov/?id=EJ1046635. 


\section{Meta-Análisis del Artículo}




\section{Dimensión Cuantitativa}

\section{Perfil de Evaluación entre pares}
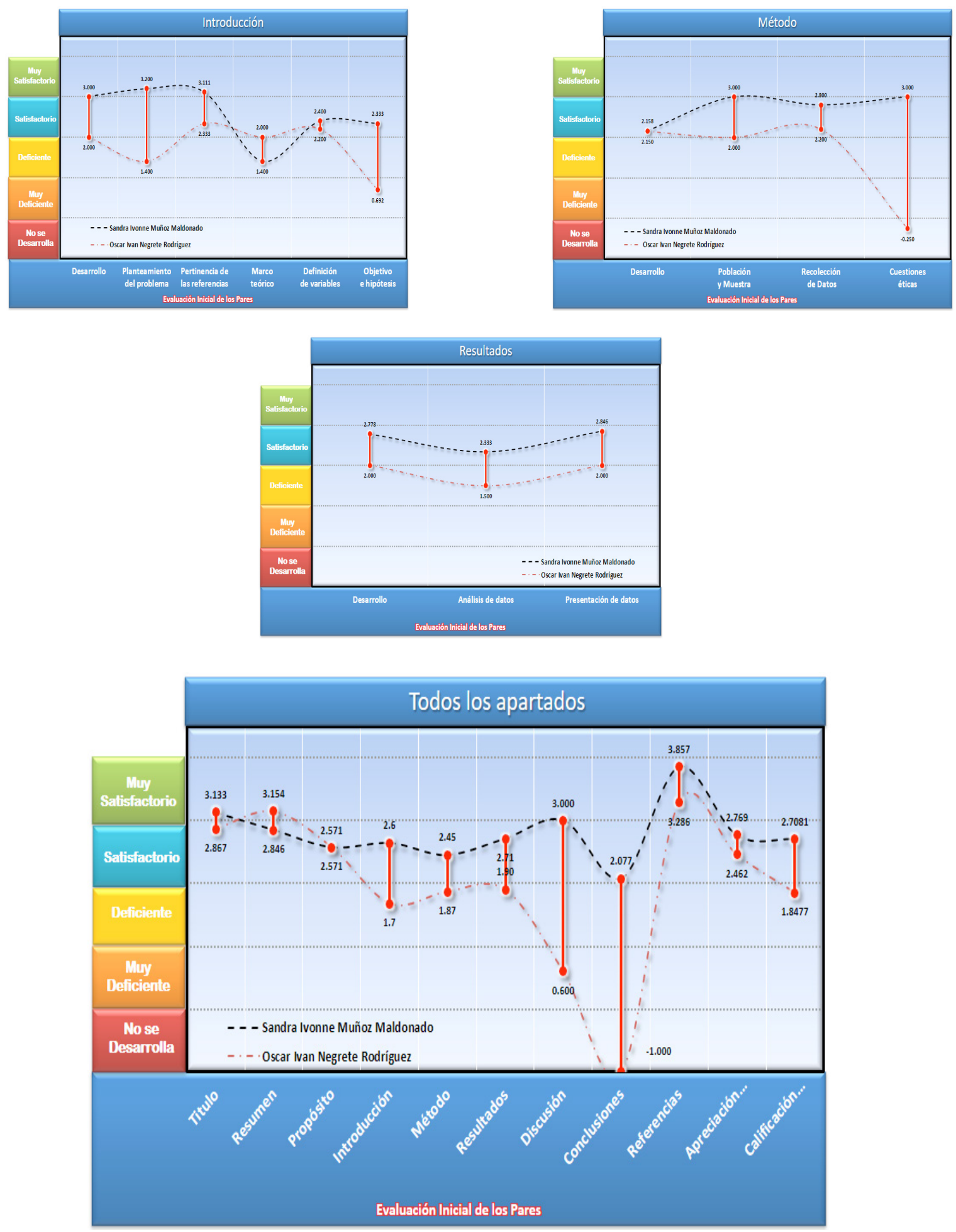


\section{Índice de Concordancia}

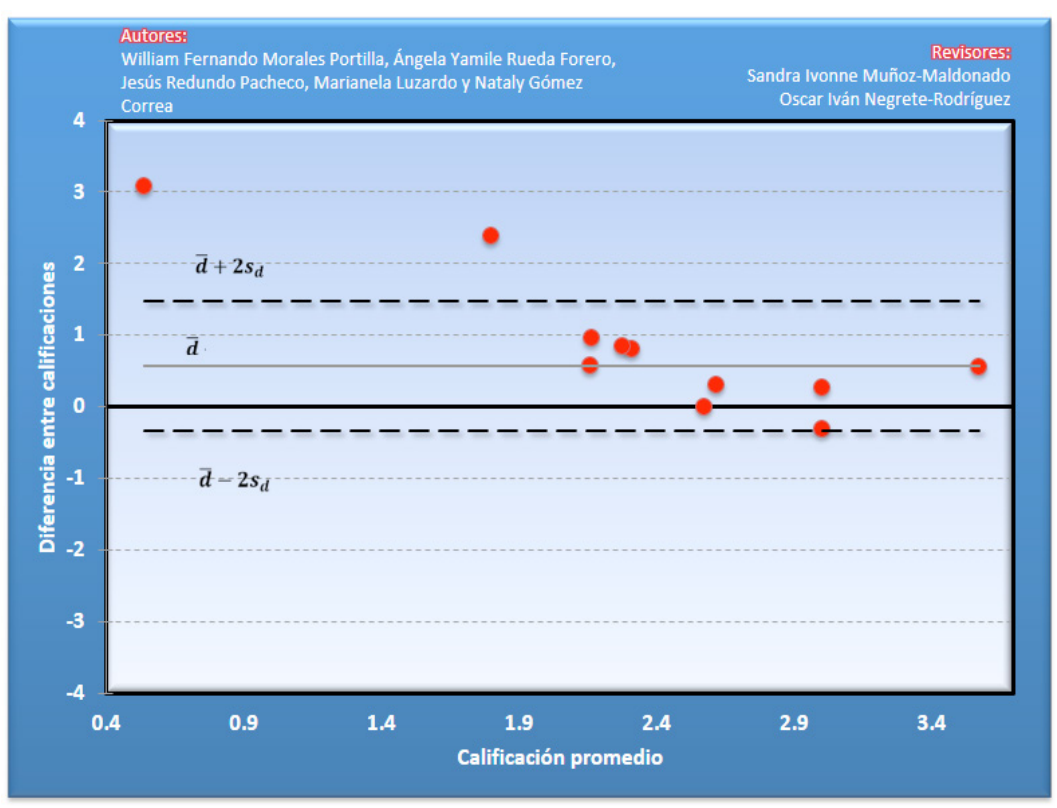

Índice de Acuerdo

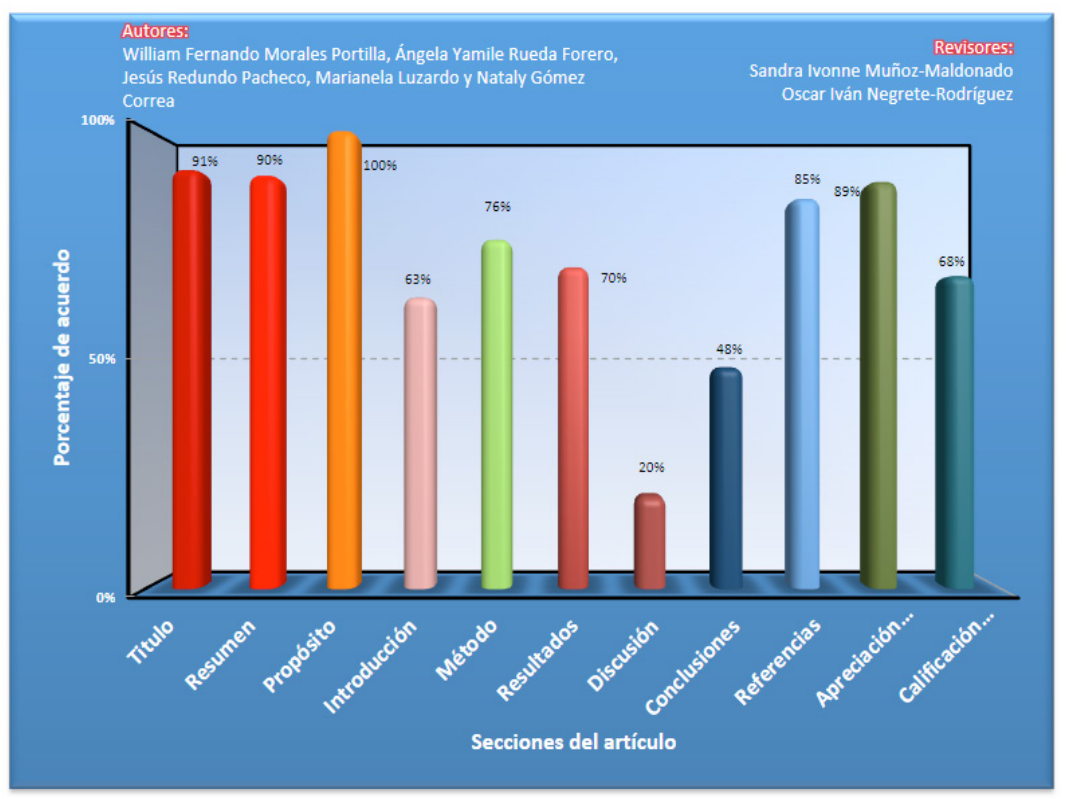




\begin{tabular}{|l|}
\hline \multicolumn{1}{|c|}{ Revisor 1 } \\
\hline \multicolumn{1}{|c|}{ Oscar Ivan Negrete Rodríguez } \\
\hline \multicolumn{1}{|c|}{ Título/A } \\
\hline $\begin{array}{l}\text { De acuerdo a las variables que miden podría ser más } \\
\text { específico el título. }\end{array}$
\end{tabular}

\section{Resumen}

El título no explica en dónde o el tipo de población para ser más especifico, además de que no evidencia los objetivos y aspectos metodológicos en donde se buscan correlaciones entre los factores evaluados, por ejemplo "Factores personales y socioeconómicos relacionados con cyberbullying en adolescentes colombianos"

Convendría precisar los coeficientes de correlación en-
contrados. La redacción en inglés no es del todo correc-
ta, pareciera que se hizo una traducción textual de las
palabras empleadas al ingles..

En el resumen no se indica el tipo de estudio, diseño de investigación, se emplean abreviaturas para TICs y los títulos de los inventarios de personalidad, en los resultados no se menciona la relación de los factores de personalidad con el cyberbullying y las conclusiones no dan respuesta al objetivo más bien resaltan lo que se debe hacer en la investigación, pero no concluyen con una idea derivada de los resultados y el objetivo.

\section{Próposito del Estudio}

Convendría modificar la redacción del objetivo, porque aunque se empieza hablando de cyberbullying se da por hecho que el lector deducirá que se refieren al cyberbullying al usar la frase "este fenómeno", también podría eliminar las palabras "si variables como..." cuando son específicamente esas variables las que analizan en la investigación.

\section{Introducción}

No es clara la redacción de los objetivos, deben enunciarse de manera puntual la relación (correlación) que se anticipa se presentará entre las variables del estudio. No presentan hipótesis.
No se plantean las preguntas de investigación como tal, se menciona el objetivo y de ahí se deduce la pregunta, pero no está explícita, asimismo no se deja claro cómo se contestarán las preguntas de investigación
No se denotan las preguntas de investigación hipótesis a contrastar y no se explica un modelo teórico que sustente la investigación y el objetivo se centra en identificar factores de riesgo y no si existe relación entre las variables, tomando en cuenta que una simple correlación no explica que sea un factor de riesgo 


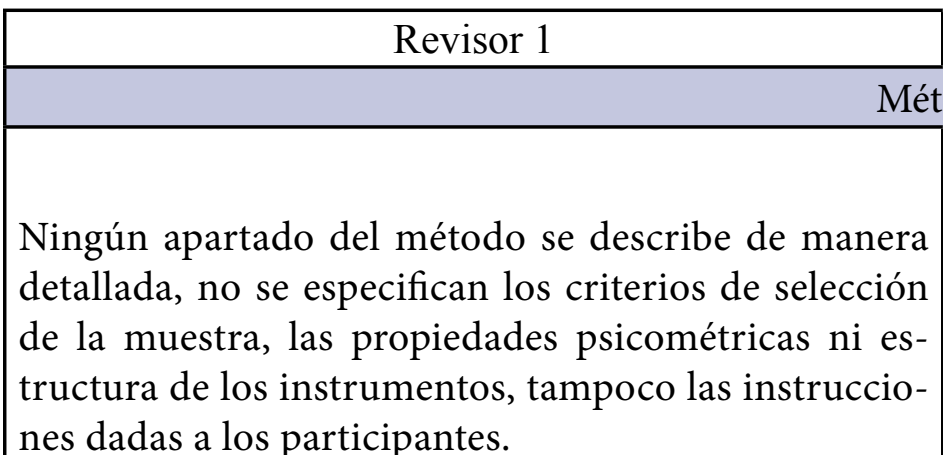

\section{Resultados}

No se describe un tipo de estudio ni diseño de investigación sólo se menciona un enfoque cuantitativo, lo cual no da respuesta a los objetivos y no va en relación a los resultados y las hipótesis que se marcan, no de definen las variables operacionalmente, no se describen los análisis estadísticos realizados, para que estén en conjunción con las hipótesis

Se presenta sólo un listado de datos estadísticos, descriptivos e inferenciales, sin ir articulando la descripción y análisis de los resultados obtenidos. Deberían describir el tipo de correlación que fue empleada (Rho, Tau b, R).
Como desde un inicio no se incluyeron las hipótesis la redacción de los resultados no sigue un orden de las preguntas de investigación, hipótesis u objetivos, se recomienda incluir éstas desde la introducción para en este apartado irle dando respuesta a cada una y llevar un orden lógico

\section{Discusión}

Se hace un contraste muy general de los resultados del estudio con la revisión de la literatura sin arriesgar a estables las explicaciones tentativas de las diferencias y semejanzas encontradas.
En las conclusiones se describen rasgos psicológicos que no empatan con los evaluados y por lo tanto no pueden ser comparados, ya que no se evalúa autoconcepto, neuroticismo o psicotisismo, por lo lo que debería contrastarse con estudios en donde se evaluaran las mismas variables psicológicas.

\section{Conclusiones}

No se hace un apartado de conclusiones como tal y no se retoman limitaciones del estudio e investigaciones futuras que puedan dar mejor respuesta a la pregunta de investigación, deja muy corto el aspecto de los factores psicológicos, ya que no se explica cómo es que los factores correlacionados tanto con la víctima o agresor pueden ayudar a explicar el comportamiento de ciberacoso

\section{Referencias}

Convendría incrementar el número de referencias de los últimos 5 años 
